\title{
P04-3-8
}

Poster session

\section{Correlation of Body Weight and Bone Mineral Content with Bone Biomarkers in Thai Obese Boys and Girls}

\section{Rapheeporn Khwanchuea, Chuchard Punsawad}

School of Medicine, Walailak University, Nakhonsithammarat, Thailland

Objective This study aimed to investigate correlations of anthropometrics, body composition, bone parameters of the lumbar spine and whole body, and bone markers in non-obese and obese Thai boys and girls.

Methods This study was performed in 35 boys and 31 girls aged $13.00 \& \# 61617 ; 0.00$ and $13.48 \& \# 61617 ; 0.10$ years enrolled in secondary schools in southern Thailand. Subjects were grouped into control group (normal-to-overweight) (BMI \&\#61500; 25) and obesity (BMI $>=27.5$ ). Anthropometric indices for obesity and body composition (BW, BMI, LBM, BFM, \%fat, and \%lean) were recorded. Bone parameters (BMC, BMD, and Z-scores) of the lumbar spine (L1-L4) and whole body were assessed by DXA. Levels of bone formation markers (BALP, and OSC) and bone resorption markers (CTX), were measured using the methods of ELISA. Correlations between those parameters, and bone biomarkers were evaluated and compared between control and obese groups of boys and girls.

Results In both obese boy and girl subjects have higher whole body BMC than the control groups $(\mathrm{p}<0.01)$. Levels of bone biomarkers were not different between control and obese groups of boys and girls, however those of the obese boys were higher than the obese girls $(\mathrm{p}<0.05)$. In obese girl subjects, only BW was negative correlations with CTX and BALP, whereas a positive correlation with OSC, and BMC of the lumbar spine and whole body were negative correlation with OSC $(\mathrm{p}<0.05)$. However, these association were not found in the obese boy subjects.

Conclusions BW was a negative association with CTX and BALP, and BMC of both lumbar spine and whole body was a negative correlation with OSC particularly in the obese girl subjects. 